Rapid Reviews COVID-19

\title{
Review 1: "Vaccination for some childhood diseases may impact the outcome of covid-19 infections"
}

\section{Masako Kinoshita ${ }^{1}$}

${ }^{1}$ National Hospital Organization Utano National Hospital

Published on: Jan 28, 2022

License: Creative Commons Attribution 4.0 International License (CC-BY 4.0). 


\section{$\underline{\text { RR:C19 Evidence Scale rating by reviewer: }}$}

- Potentially informative. The main claims made are not strongly justified by the methods and data, but may yield some insight. The results and conclusions of the study may resemble those from the hypothetical ideal study, but there is substantial room for doubt. Decision-makers should consider this evidence only with a thorough understanding of its weaknesses, alongside other evidence and theory. Decisionmakers should not consider this actionable, unless the weaknesses are clearly understood and there is other theory and evidence to further support it.

******************************************

\section{Review:}

Gobe et al. analysed the impact of existing immunization on COVID-19 outcome and found that mean percentage death rates were lower in countries that vaccinated for hepatitis-B birth dose, Bacille Calmette-Guérin vaccine, and inactivated polio vaccine 1 st dose than those which did not report vaccination. The effect of vaccination was different between high and low-income countries. The authors conclude the potential benefit of vaccines on improving COVID-19 outcomes. The theme of this study is important; however, there are several concerns that should be addressed before publication.

\#1. The authors used variables of ratios of COVID-19 deaths and recoveries in each country. Considering that infant BCG vaccination plays a protective role against mass infection by reducing silent spreaders, i.e., asymptomatic or mild cases that can disseminate of SARS-CoV-2, as well as reducing the severity, local or nationwide COVID-19 prevalence should be analyzed at first. This shortcoming of study design has brought about the paradoxical findings that percent recoveries were significantly higher in non-vaccinating countries for PCV, RotaCV, and Hepatitis B-3 vaccines, as described in the Discussion.

\#2. In this study, group comparison and correlation analysis are performed based on the types of vaccine. Since several types of vaccinations are performed in each country, confounding factors should be corrected. Additional analysis using multivariate regression analysis would be informative. Parametric methods were employed in the statistical analysis of this study, but normality assumptions should be tested to ensure their suitability. 
\#3. The protective mechanism of the BCG vaccine against COVID-19 is attributed to trained immunity in accord with the previous literature. As for other types of vaccines, the authors should provide a rationale of how those vaccines exert the protection against COVID-19, and whether or not their mechanisms are similar to that of BCG which has been almost established.

\#4. Analysis by economic level of countries showed that Pol-3 correlated with a significant unfavorable outcome in high-income countries and that YFV negatively correlated with percent recovery in low-income countries. These contradictory findings are possibly associated with a local high prevalence requiring immunization of these diseases. Further investigation of characteristics of included countries would explain the reason for the inconsistent findings.

\#5. The manuscript's title: 'Vaccination for some childhood diseases...' is misleading because the diseases are not limited to childhood but can occur at any age. In addition, COVID-19 vaccines are already emerging. Thus phrases indicating pre-existing vaccines inoculated in the neonatal period, infancy, or childhood should be modified in order to avoid confusion.

\#6. The novel findings of this study should be clarified. In the current form, this study only supports previous findings of the protective role of BCG against COVID-19.

\#7. The reference list needs to be updated especially there are emerging publications on the relationship between BCG vaccination and COVID-19.

\#8. The authors should provide a rationale for confounding factors raised in Study limitations based on literature. 